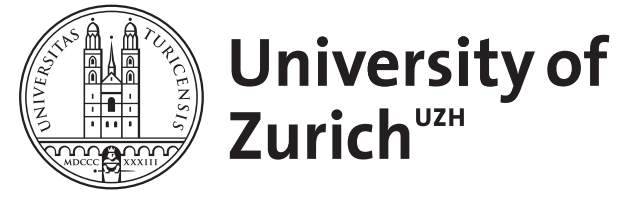

Zurich Open Repository and Archive

University of Zurich

University Library

Strickhofstrasse 39

CH-8057 Zurich

www.zora.uzh.ch

Year: 1959

\title{
Thermokraftmessungen am Silberjodid
}

Wagener, K

DOI: https://doi.org/10.1524/zpch.1959.21.1_2.074

Posted at the Zurich Open Repository and Archive, University of Zurich

ZORA URL: https://doi.org/10.5167/uzh-154581

Journal Article

Published Version

Originally published at:

Wagener, K (1959). Thermokraftmessungen am Silberjodid. Zeitschrift für Physikalische Chemie, 21(12) : $74-81$.

DOI: https://doi.org/10.1524/zpch.1959.21.1_2.074 


\title{
Thermokraftmessungen am Silberjodid
}

\author{
Von
}

\section{K. WAGENER}

Mit 3 Abbildungen im Text

(Eingegangen am 24. 2. 59)

\section{Einleitung}

Zur näheren Kenntnis der Fehlordnungserscheinungen und der Elektrizitätsleitung im $\alpha-A g J$ unter höheren Joddampfdrucken wurden Thermokraftmessungen ausgeführt. Das Interesse hierfür ergab sich, als neuere Messungen von $\mathrm{WeIss}^{1}$ am $\alpha-A g J$ eine Leitfähigkeitserhöhung bis zu $30 \%$ unter der Einwirkung von Joddampf zeigten. Diese Leitfähigkeitszunahme wurde auf die Bildung von Defektelektronen zurückgeführt, die bei der über das stöchiometrische Verhältnis hinausgehenden Aufnahme von Jod nach der Gleichung

$$
J_{2}(\text { Gas }) \rightleftarrows 2 J \text { (chemisorb.) } \rightleftarrows 2 J^{-}+2 \oplus \text { (Kristall) }
$$

gebildet werden. Andererseits ist bekannt, daß AgJ unter der Einwirkung von Joddampf wägbare Mengen von Jod aufnimmt, und es erscheint nicht ausgeschlossen, daß als Folge davon sich auch die Kationenbeweglichkeit ändert. Eine beobachtete Leitfähigkeitsänderung wäre dann nicht mehr eindeutig einer Änderung der Defektelektronendichte zuzuschreiben.

Sind die WeIssschen Messungen (bezüglich der Elektronenüberführungszahl in Jodatmosphäre) quantitativ richtig, so sollte sich diese Tatsache auch in beobachtbarer Weise in der thermo-chemischen Bilanz, wie sie von REINHOLd für feste elektrolytische Ketten aufgestellt wurde, anzeigen.

${ }^{1} \mathrm{~K}$. WeIss, Dissertation Göttingen 1956. S. 66 und 98. 
Nach REINhold ${ }^{2}$ läßt sich der Temperaturkoeffizient der EMK eines galvanischen Elements durch die Differenz der Thermokräfte darstellen, die die aus den Elektroden und dem Elektrolyten des Elements gebildeten Thermoketten besitzen:

wobei

$$
\frac{d \mathrm{EMK}}{d T}=\frac{d E_{2}}{d T}-\frac{d E_{1}}{d T},
$$

$d$ EMK den Temperaturkoeffizienten der isothermen Kette

${ }_{d T} \quad M e / M e X / X_{2}$,

$\frac{d E_{2}}{d T} \quad$ die Thermokraft der Kette $X_{2} / M e X / X_{2}$ und

$\frac{d E_{1}}{d T^{-}} \quad$ die Thermokraft der Kette $M e / M e X / M e$

bedeuten. Für $\mathrm{AgCl}$ und $\mathrm{AgBr}$ sind die erforderlichen Messungen von REINHOLD selbst ausgeführt worden ${ }^{2,3}$, wobei sich im Rahmen der Meßgenauigkeit gute Übereinstimmung nach (I) ergab. Für $A g J$ wurde von Reinhold nur die Thermokette $A g / A g J / A g^{3}$ und die Temperaturabhängigkeit der EMK der Bildungskette $A g / A g J / J_{2}$ (Graphit) ${ }^{4}$ gemessen, so daß eine Nachprüfung nach (I) bisher nicht möglich war.

\section{Herstellung der Meßproben, Durchführung und Ergebnisse der Messungen}

Die Thermokraftproben wurden in folgender Weise hergestellt: als Elektroden dienten Platindrähte von $0,5 \mathrm{~mm}$ Stärke, die an den kontaktseitigen Enden auf einer Länge von etwa $1,5 \mathrm{~cm}$ zu einer $0,1 \mathrm{~mm}$ dicken und 2 bis $3 \mathrm{~mm}$ breiten Folie ausgewalzt wurden. Diese ausgewalzten Enden wurden alsdann zu einer in der Längsrichtung verlaufenden Rinne gefalzt. Nach sorgfältigem Reinigen und Ausglühen der so vorbereiteten Elektroden wurde in ihnen so viel von dem später verwendeten Silberdraht geschmolzen, daß sie etwa zur Hälfte gefüllt waren. In diese Schmelze wurde dann das Ende des später zu jodierenden Drahtes eingetaucht und das Ganze zum Erstarren gebracht. Diese Silber-Platin-Kontaktierung erwies sich auch nach dem Jodieren als sehr zuverlässig und mechanisch äußerst haltbar.

Die Silberdrähte der Proben hatten eine Länge von 10 bis $15 \mathrm{~cm}$ und einen Durchmesser von 0,3 mm. Der Silberdraht wurde von der

${ }^{2}$ H. ReInhold, Z. Elektrochem. angew. physik. Chem. 39 (1933) 290. 
Firma W. C. Heraeus, Hanau, bezogen und hatte eine Mindestreinheit von $99,99 \%$.

Die so hergestellte $P t$ - $A g$-Pt-Kette wurde nun in ein T-förmiges Glasrohr eingeschmolzen, dessen rechtwinklig abstehender Schenkel zur Aufnahme des Jodvorrats diente. Das Probengefäß wurde mit einer hinreichenden Menge (doppelt sublimierten) Jodes beschickt und mit dem noch offenen Ende über eine Kühlfalle an eine Hochvakuumanlage angeschlossen. Der erreichbare Dampfdruck war durch den Joddampfdruck bei Zimmertemperatur vorgegeben. Es wurde nun etwa 20 Minuten evakuiert, wobei natürlich ein Teil des Jods herausgepumpt, aber gleichzeitig der Sauerstoffpartialdruck ständig vermindert wurde.

Das so unter Vakuum abgeschmolzene Probengefäß zeigte - wenn das Jod bei Eistemperatur kondensiert wurde - ein kaum mehr sichtbares Glimmleuchten im Hochfrequenzfeld, was einem Dampfdruck von etwa $10^{-2}$ Torr (dem Joddampfdruck bei $0^{\circ} \mathrm{C}$ ) entspricht. Der restliche Sauerstoffpartialdruck muß also in jedem Fall kleiner als $10^{-2}$ Torr gewesen sein.

Über jeden Schenkel wurde ein dickwandiger Kupferrohrofen geschoben. Die Enden und das Kreuzstück des Rohres wurden mit Glaswatte und Asbestkordel abgedichtet. Die Temperaturdifferenz $\Delta T$ zwischen den Kontakten wurde mit einem Differentialthermoelement bestimmt.

Der $A g J$-Faden erwies sich trotz seiner beträchtlichen Länge und seinem geringen Querschnitt als äußerst haltbar. Mit einer Probe wurde mehrere Stunden bei $520^{\circ} \mathrm{C}$ gemessen (Smp. AgJ $555^{\circ} \mathrm{C}$ ), ohne daß er zerstört wurde. Einige Proben sind nach etlichen Messungen aus- und später wieder eingebaut worden, wobei sie wieder genau die alten Meßwerte ergaben. Die gute Übereinstimmung der an mehreren Proben gemessenen Werte sei besonders erwähnt.

Der Widerstand der Thermokette lag in der $\alpha$-Phase zwischen $10^{3}$ und $10^{4} \mathrm{Ohm}$, in der $\beta$-Phase zwischen $10^{7}$ und $10^{8} \mathrm{Ohm}$. Die Thermospannungen wurden mit einem Kompensator gemessen und konnten in der $\alpha$-Phase mühelos mit einer Genauigkeit von $10^{-4}$ ihres Betrages bestimmt werden. In der $\beta$-Phase lag die Genauigkeit bei etwa $1 \%$. Am unsichersten sind die Messungen in der $\beta$-Phase bei tiefen Temperaturen $\left(110^{\circ} \mathrm{C}\right)$ und besonders bei geringem Joddruck. Diese Werte können um mehrere Prozent falsch sein. Die Messungen in der $\beta$-Phase dienten nur zur Orientierung und werden bei der weiteren Auswertung 
nicht berücksichtigt. Die Stromempfindlichkeit des bei den Messungen in $\operatorname{der} \beta$-Phase verwandten Nullinstruments betrug $10^{-11} \mathrm{Amp} / \mathrm{mm} \cdot \mathrm{m}$.

Einen Eindruck von der Meßgenauigkeit gibt das Beispiel in Abb. 1. Die Abhängigkeit der Thermokraft von der mittleren Probentemperatur

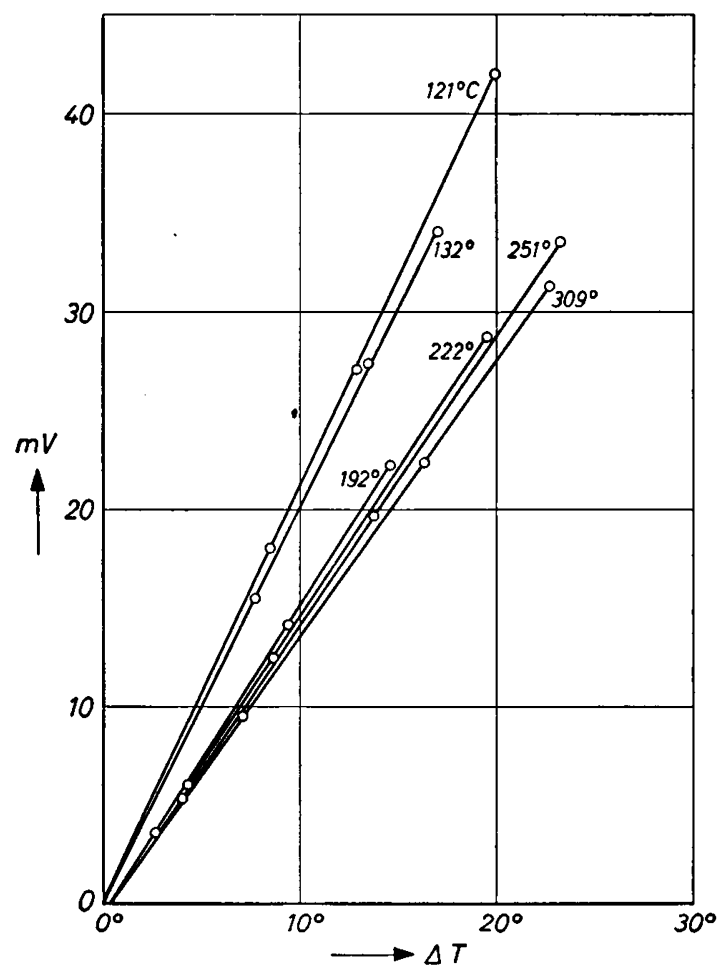

Abb. 1. Thermokraft der Kette $P t / A g J / P t$ bei einem Joddampfdruck von 0,03 Torr. Die Temperaturen sind die mittleren Probentemperaturen. Der gesuchte Anstieg der Kurven wurde ermittelt durch Berechnung der ,,besten Geraden" durch die Meßpunkte nach der Methode der kleinsten Fehlerquadratsumme

und dem Joddampfdruck zeigen die Abb.2 und 3. Stets war die heiße Elektrode negativ, d. h. das Vorzeichen der Thermokraft ist nach der üblichen Schreibweise negativ.

Besondere Aufmerksamkeit wurde bei der Messung darauf gerichtet, ob Hystereseerscheinungen oder Hemmungen auftraten. Das ist mit Sicherheit nicht der Fall. Manche Meßpunkte haben unter konstanten Bedingungen 10 bis 16 Stunden gestanden, ohne sich zu ver- 
ändern. Die Thermospannung stellte sich in der $\alpha$-Phase praktisch unmittelbar ein. $\mathrm{Zu}$ beachten ist nur die langsame Gleichgewichtsvereinstellung der Reaktion $J_{2}$ (Gas) $\rightleftarrows 2 J$ (Kristall) in der $\beta$-Phase. Das System wurde daher vor Beginn der Messung mehrere Stunden (oft über Nacht) stehen gelassen.

Gemessen wurde grundsätzlich nur, wenn alle beobachteten Größen zeitlich völlig konstant waren.

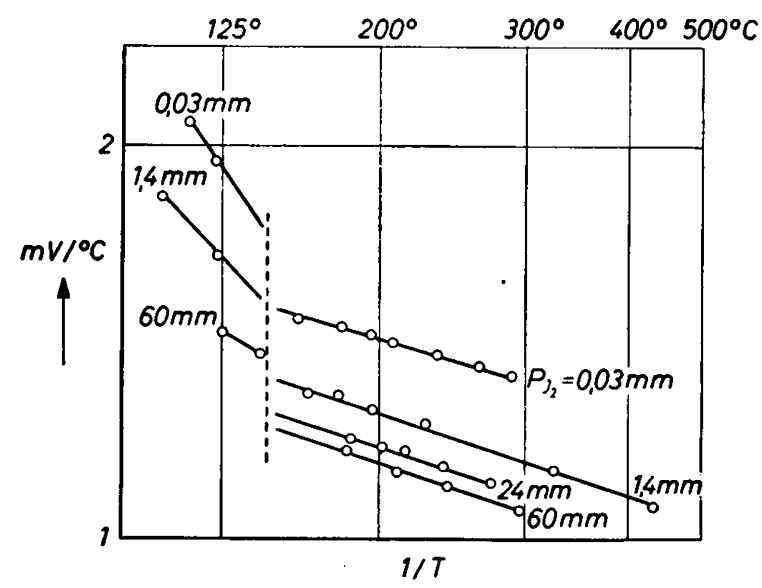

Abb.2. Abhängigkeit der Thermokraft von der mittleren Probentemperatur bei verschiedenen Joddampfdrucken

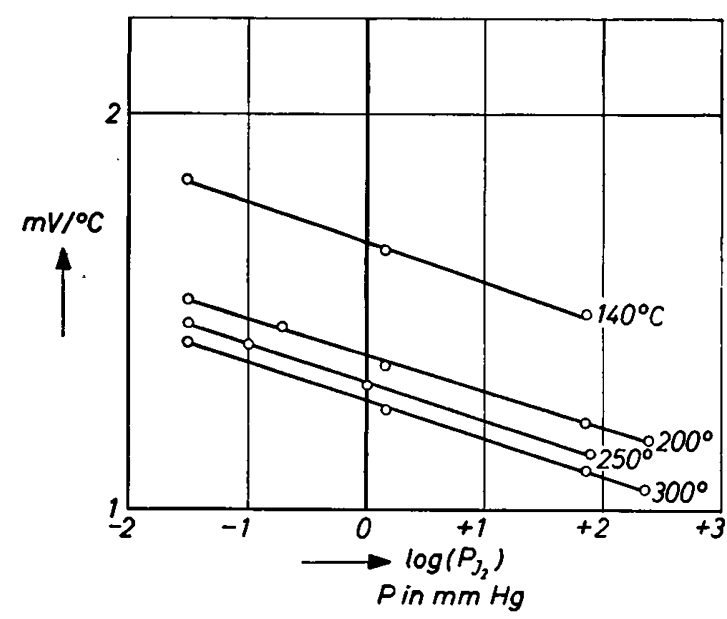

Abb. 3. Abhängigkeit der Thermokraft vom Joddampfdruck für vier verschiedene Temperaturen 
Die Thermokraft des Metalls (Platin) ist um Größenordnungen kleiner als die des Salzes, so daß eine (an sich mögliche) Korrektur unnötig ist.

Numerische Ergebnisse aus den Messungen:

Tabelle 1. Abhängigkeit der Thermokraft vom Joddampfdruck (aus den Messungen $\operatorname{der} A b b .3)$

\begin{tabular}{c|l|l}
\hline${ }^{\circ} \mathrm{C}$ & \multicolumn{1}{|c|}{$d E_{2} / d \ln P_{2}$} & $\begin{array}{c}-(F / R)\left(d E_{2} / d\right. \\
\left.\ln P_{J_{2}}\right)\end{array}$ \\
\hline $200^{\circ}$ & $4,3 \cdot 10^{-5} \mathrm{~V} / \mathrm{grad}$ & $1 / 2$ \\
$250^{\circ}$ & 4,4 & $1 / 1,95$ \\
$300^{\circ}$ & 4,5 & $1 / 1,9$
\end{tabular}

\section{Diskussion der Meßergebnisse und Prüfung der ReINHoLdschen Beziehung}

Zunächst ist die Präzision der Potentialeinstellung an der Thermokette und die ausgezeichnete Reproduzierbarkeit der Ergebnisse hervorzuheben. Wie Tab. 1 zeigt, ist die zu erwartende Quadratwurzelbeziehung zwischen Thermokraft und Jodpartialdruck gut erfüllt.

Zur Prüfung der Beziehung (I) seien die Ergebnisse von Reinhold mitgeteilt. Die Thermokraft der Kette $A g / A g J / A g$ fand er $^{3}$ zwischen 420 und $600^{\circ} \mathrm{K}$ temperaturunabhängig zu

$$
d E_{1} / d T=-0,56 \cdot 10^{-3} \mathrm{~V} /{ }^{\circ} \mathrm{K} .
$$

Als Temperaturkoeffizienten der EMK der Bildungskette $A g|A g J|$ $J_{2}(C)$ ergibt sich aus seinen Messungen ${ }^{4}$

$$
d \mathrm{EMK} / d T=0,12 \cdot 10^{-3} \mathrm{~V} /{ }^{\circ} \mathrm{K} \quad\left(\text { für } P_{J_{z}}=1 \mathrm{~atm}\right) .
$$

Der Temperaturkoeffizient wurde auf die verwendeten Jodpartialdrucke umgerechnet uach

$$
\begin{aligned}
\operatorname{EMK}\left(T, P_{J_{2}}\right) & =\mathrm{EMK}_{\text {Reinh }}^{\circ}+(R T / 2 F) \ln P_{J_{s}} ; \\
d \mathrm{EMK} / d T & =\left(0,12+0,0992 \log P_{J_{2}}\right) 10^{-3} \mathrm{~V} /{ }^{\circ} \mathrm{K} .
\end{aligned}
$$

Ein erster Vergleich dieser Werte (Spalte f, Tab.2) mit den Meßergebnissen beider Thermoketten zeigt eine erhebliche Diskrepanz. Man darf jedoch aus diesem Ergebnis keine voreiligen Schlüsse ziehen. 
Wie nämlich eine kritische Betrachtung zeigt (diesen Hinweis verdanke ich Herrn Prof. Dr. C. WAgner), sind die Reinholdschen EMKMessungen an der Bildungskette keineswegs unter so definierten Bedingungen durchgeführt, daß man ihnen noch den Temperaturgang mit Sicherheit entnehmen könnte.

In der Tat liefert eine Berechnung des Temperaturkoeffizienten einen Wert, der sich nach Betrag und Vorzeichen von dem unterscheidet, der sich aus den ReInHoldschen Messungen ergibt. Die Genauigkeit der den Berechnungen zugrunde gelegten Entropiedaten dürfte die hier gestellten Forderungen noch überschreiten.

Mit Vorzeichen nach der Stockholm Convention ergibt sich (der Index ${ }^{\circ}$ gilt für $P_{J_{2}}=1 \mathrm{~atm}$ )

$$
\begin{aligned}
\mathrm{EMK}^{\circ} & =-\frac{1}{F} \cdot \Delta G^{\circ} \\
\frac{d \mathrm{EMK}^{\circ}}{d T} & =-\frac{1}{F} \cdot \frac{d \Delta G^{\circ}}{d T}=+\frac{\Delta S^{\circ}}{F} \\
\frac{d \mathrm{EMK}}{d T}\left(P_{J_{2}}\right) & =-\left[\frac{d \mathrm{EMK}^{\circ}}{d T}(T)+0,992 \cdot 10^{-4} \log P_{J 2}\right] \frac{V}{{ }^{\circ} K} .
\end{aligned}
$$

Für die numerische Berechnung von $\Delta S^{\circ}$ wurden die Daten aus National Bureau of Standards Circular 500 und aus K. K. KeLLeY, Bureau of Mines Bulletin 476 (Washington 1949) verwendet. Die so berechneten Werte von $d \mathrm{EMK} / d T$ (Spalte g in Tab.2) zeigen gute Übereinstimmung mit den Meßwerten anhand der REINHOLDschen Beziehung (I). Die verbleibenden kleinen Differenzen (Spalte $h$ in Tab. 2) gehen mit wachsender Temperatur gegen Null. Dieser Befund entspricht ganz den Erfahrungen bei $\mathrm{AgCl}$ und $\mathrm{AgBr}$. Die Absolutbeträge der Abweichungen sind kleiner als bei $\mathrm{AgCl}$ und insbesondere $A g B r$. Es tritt auch kein Gang der Abweichungen in Abhängigkeit vom Joddampfdruck auf.

Nach diesem Ergebnis dürfte selbst unter höheren Joddampfdrucken zumindest kein mit der Ionenleitung vergleichbarer elektronischer Leitungsanteil vorliegen, wieihn die WeIssschen Messungen scheinbar anzeigten; denn die Kette $A g / A g J / A g$ hat - wieÜberführungsmessungen zeigen - keinen elektronischen Leitungsanteil und die Größe $d$ EMK $/ d T$ ist ebenfalls unter der Voraussetzung reiner Ionenleitung berechnet worden. 
Tabelle 2. Zusammenstellung der Ergebnisse und Vergleich nach der REINHOLDschen Beziehung

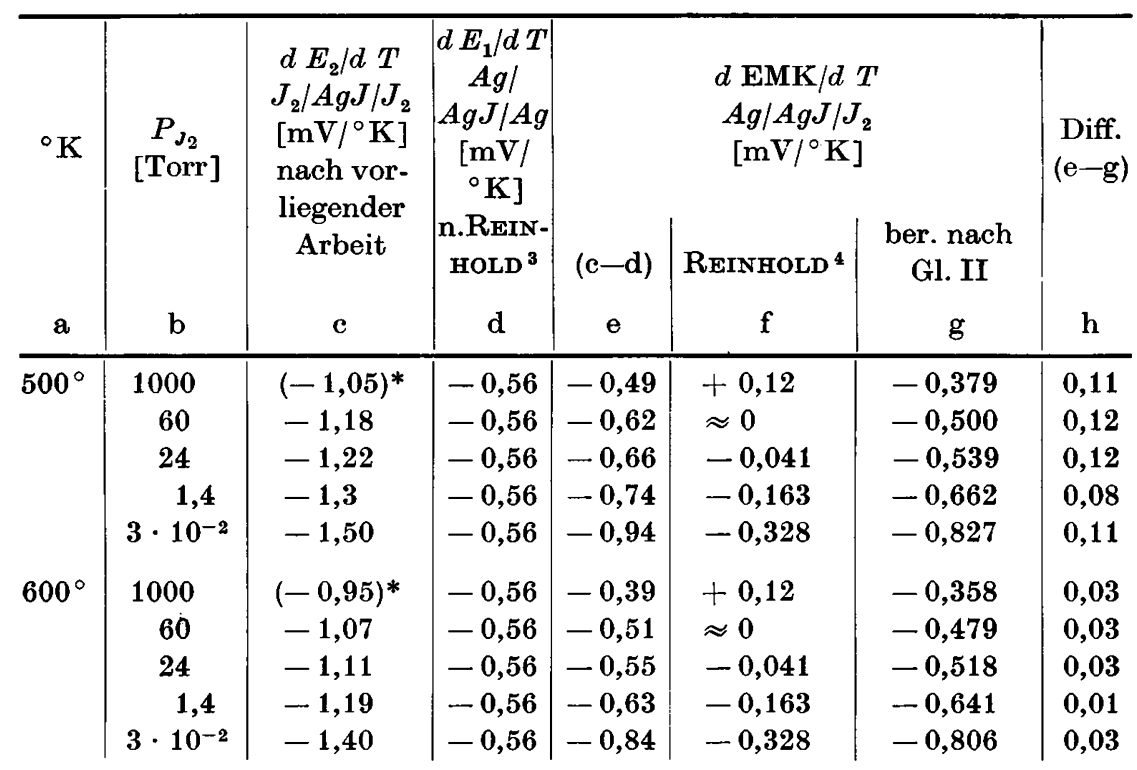

* Aus den Meßwerten extrapoliert.

Herrn Prof. Dr. W. Jost danke ich sehr für sein Interesse an dieser Arbeit. Herzlicher Dank gilt auch Herrn Prof. Dr. C. WAGNer für wertvolle Hinweise zur Auswertung der Messungen.

Physikalisch-Chemisches Institut der Universität Zürich

${ }^{3}$ H. Reinhold, Z. anorg. allg. Chem. 171 (1928) 181.

4 H. Rennhold, Z. Elektrochem. angew. physik. Chem. 40 (1934) $361-364$. 